\title{
Dissecting Symptomatology and Fumonisin Contamination Produced by Fusarium verticillioides in Maize Ears
}

\author{
Laura Morales, ${ }^{\dagger}$ Thiago P. Marino, Anthony J. Wenndt, Julia Q. Fouts, James B. Holland, and Rebecca J. Nelson
}

First, third, fourth, and sixth authors: School of Integrative Plant Science, Cornell University, Ithaca, NY; second and fifth authors: Department of Crop and Soil Sciences, North Carolina State University, Raleigh; and fifth author: Plant Science Research Unit, United States Department of Agriculture-Agricultural Research Service, Raleigh, NC.

Accepted for publication 6 July 2018.

\begin{abstract}
The fungus Fusarium verticillioides can infect maize ears, contaminating the grain with mycotoxins, including fumonisins. This global public health threat can be managed by breeding maize varieties that are resistant to colonization by $F$. verticillioides and by sorting grain after harvest to reduce fumonisin levels in food systems. Here, we employed two $F$. verticillioides inoculation techniques representing distinct infection pathways to dissect ear symptomatology and morphological resistance mechanisms in a diverse panel of maize inbred lines. The "point" method involved penetrating the ear with a spore-coated toothpick and the "inundative" method introduced a liquid spore suspension under the husk of the ear. We evaluated quantitative and qualitative indicators of external and internal symptom severity as lowcost proxies for fumonisin contamination, and found that kernel bulk

density was predictive of fumonisin levels (78 to $84 \%$ sensitivity; 97 to $99 \%$ specificity). Inundative inoculation resulted in greater disease severity and fumonisin contamination than point inoculation. We also found that the two inoculation methods implicated different ear tissues in defense, with cob morphology being a more important component of resistance under point inoculation. Across both inoculation methods, traits related to cob size were positively associated with disease severity and fumonisin content. Our work demonstrates that (i) the use of diverse modes of inoculation is necessary for combining complementary mechanisms of genetic resistance, (ii) kernel bulk density can be used effectively as a proxy for fumonisin levels, and (iii) trade-offs may exist between yield potential and resistance to fumonisin contamination.
\end{abstract}

Fusarium verticillioides is a fungal pathogen that causes Fusarium ear rot (FER) of maize (Zea mays L.) and contaminates grain with fumonisins (FUM), a family of mycotoxins produced by Fusarium spp. (Rheeder et al. 2002). FER can decrease yields and FUM contamination reduces the marketability of maize grain (Mueller et al. 2016). In addition, the serious health risks associated with exposure to mycotoxins, including FUM, (Wild and Gong 2009) have led the World Health Organization to call for action against mycotoxin contamination in the developing world (Wild et al. 2015). Not only are the tropical and subtropical environments characteristic of developing nations conducive to $F$. verticillioides infection and subsequent FUM production in maize grain (Mutiga et al. 2014; Parsons and Munkvold 2012; Schjøth et al. 2009) but also food systems in these regions often provide minimal regulation of mycotoxins; the combination of these factors with the role of maize as a staple crop has led to high levels of FUM exposure in the developing world (Kimanya et al. 2010; Mutiga et al. 2014).

Although FUM cannot be completely removed from foodstuffs, pre- and postharvest interventions can reduce FUM levels. Preharvest strategies include employing sound agronomic practices (Ariño et al. 2009; Munkvold 2003) and more long-term approaches such as breeding for resistance in maize varieties (Lanubile et al. 2017). Genetic resistance to FUM contamination is moderately to highly heritable and quantitatively controlled (Bolduan et al. 2009;

†Corresponding author: Laura Morales; E-mail: 1m596@cornell.edu

Funding: Support was provided by the North Carolina Corn Growers Association (grant 15-09).

*The $\boldsymbol{e}$-Xtra logo stands for "electronic extra" and indicates that one supplementary dataset is published online.

This article is in the public domain and not copyrightable. It may be freely reprinted with customary crediting of the source. The American Phytopathological Society, 2018.
Eller et al. 2008; Presello et al. 2007; Robertson-Hoyt et al. 2006). At the postharvest stage, sorting methods can remove the most toxic kernels from bulk grain samples (Afolabi et al. 2006; Stasiewicz et al. 2017), and thermochemical treatments can reduce FUM levels in maize products (Dombrink-Kurtzman et al. 2000; Humpf and Voss 2004). Practices that seek to reduce FUM contamination such as grain sorting and breeding would be facilitated by the identification of proxies that are less expensive to measure (Blandino and Reyneri 2008; Busman et al. 2012; Horne et al. 2016; Presello et al. 2007).

Spatiotemporal interactions among abiotic and biotic factors, plant development and physiology, and pathogen populations affect pathogenesis and the manifestation of FER symptoms (Mutiga et al. 2014; Parsons and Munkvold 2010, 2012). Under both natural and experimental conditions, the mode of infection influences disease outcomes. Experimental methods influence disease severity and the inferred genetic variation in resistance (Clements et al. 2003; Munkvold and Carlton 1997). Inoculation methods that represent distinct infection pathways may reveal different mechanisms of resistance. For example, "point" inoculation by penetrating the ear with a spore-coated toothpick (Lanubile et al. 2017; Zila et al. 2013) initiates infection at a single location, potentially eliciting both cob and kernel defense mechanisms and simulating infection promoted by insect damage. In contrast, "inundative" methods, involving injection of inoculum into the silk channel or underneath the husk (Lanubile et al. 2017; Robertson-Hoyt et al. 2006), may largely implicate kernel tissues in defense. To assess and combine complementary resistance mechanisms during the breeding process, it may be necessary to use diverse inoculation methods.

In this study, we used point and inundative inoculation methods to challenge a diverse panel of maize lines with $F$. verticillioides. The panel consisted of 50 maize inbred lines from the Germplasm Enhancement of Maize (GEM) project (Pollak and Salhuana 2001) and from the maize breeding program at the United States Department of Agriculture-Agricultural Research Service (USDA-ARS) 
at North Carolina State University, both of which include tropical germplasm because of its disease resistance (O'Brien et al. 2010; Pollak 2003). This experiment resulted in wide variation in kernel symptomatology. We leveraged this variation to dissect the relationships among FUM content and indicators of external and internal $F$. verticillioides infection severity, and to identify proxies for FUM contamination. In addition to FUM and FER, we measured bikaverin (BIK), a reddish Fusarium pigment that has been used as an indicator of FER (Busman et al. 2012), and kernel bulk density (KBD), which may reflect fungal degradation of the kernel tissues. We compared the severity of and the genetic variation in FUM contamination and kernel infection under the two inoculation methods. To test how point and inundative inoculation reflected kernel and cob resistance, we investigated associations between cob morphology and kernel disease severity and how these associations differed between inoculation methods.

\section{MATERIALS AND METHODS}

Field design and inoculation. Fifty maize inbred lines mostly from the USDA-ARS GEM project (Pollak and Salhuana 2001) and the maize breeding programs at North Carolina State University and the USDA-ARS at North Carolina State University were grown at the Central Crops Research Station in Clayton, NC in 2015 in an incomplete block $\alpha$ lattice design with two replicates. No seed germinated in one plot, resulting in 99 total plots. Each plot was split into two subplots by point inoculating half of the primary ears (Zila et al. 2013) and inundatively inoculating the other half (RobertsonHoyt et al. 2006). Forty-five lines had two replicates from both inoculation methods. Because of suboptimal germination, four lines (NC508, NC538, NC540, and P.3737 × NC320*3) had two inundativeand one point-inoculated replicates, and one line (CML373 $\times$ NC320*) had one replication per inoculation method. There were 194 subplots in total.

Six local $F$. verticillioides isolates (NC-36D, NC-40A, NC-40J, NC-N16, NC-N17, and NC-40N) selected for high FUM production in vitro were cultured individually on potato dextrose agar. Conidia were collected by rinsing the plates with distilled water and combined, and the mixed-isolate conidial suspension was then diluted to a final inoculum concentration of approximately $1 \times 10^{6}$ conidia $\mathrm{ml}^{-1}$. Subplots were inundatively inoculated approximately 2 weeks after silking by injecting $5 \mathrm{ml}$ of conidial suspension into the silk channel of each primary ear with a vaccinator, and another $5 \mathrm{ml}$ of inoculum was injected under the husk 1 week later. With the point method, one toothpick coated with spores from local toxigenic $F$. verticillioides isolates was inserted into the middle of each primary ear in each subplot approximately 2 weeks after silking, and the toothpick remained in the ear until harvest.

Disease phenotyping. Primary ears were harvested from each subplot at maturity, dried, then visually evaluated for FER. FER was scored based on the percentage of the kernels presenting symptoms on a 1-to-100\% scale with 5\% increments (Robertson-Hoyt et al.
2006). The average FER score of all the ears in each subplot was then calculated. In order to account for the qualitative variation in FER symptomatology, each ear was assigned a symptom type: asymptomatic, blush, starburst, purple, or moldy (Fig. 1). The asymptomatic, starburst, and moldy types have been previously described. In our study and in the literature, asymptomatic kernels had no visible external symptoms (Desjardins and Plattner 1998; Parsons and Munkvold 2010), starburst kernels were characterized by whitish streaks radiating from the stylar canal or the pedicel (Costa et al. 2003; Duncan and Howard 2010; Parsons and Munkvold 2010, 2012; Presello et al. 2008), and moldy kernels were severely degraded and exhibited matted fungal growth (Parsons and Munkvold 2010, 2012). Here, blush kernels had pink discoloration of the kernel crown, and purple kernels were degraded and had severe purplish discoloration. Each subplot was assigned a main symptom type based on the most frequent symptom type exhibited by the kernels in the subplot. Subplots with equal representation from more than one symptom type were labeled "multiple". Subplots with less than $10 \%$ average FER were considered asymptomatic.

Ears were then shelled and bulked per subplot. From each subplot, a random 250-ml volume of kernels was weighed, and KBD was calculated as the weight of the kernels divided by $250 \mathrm{ml}$. All kernels in each bulked subplot were ground into a fine powder with a Waring 7010 two-speed laboratory blender (Waring Commercial, Inc., Torrington, CT). FUM concentration was measured in duplicate from each subplot. Two 10-g subsamples from each ground subplot were placed in separate $25-\mathrm{ml}$ centrifuge tubes. To extract FUM, $20 \mathrm{ml}$ of $90 \%$ methanol was added to each $25-\mathrm{ml}$ tube, resulting in a twofold dilution factor at this step. The tubes were then shaken with a Lab-Line Environ Orbitol Shaker (Lab-Line Instruments, Inc., Melrose Park, IL) at $150 \mathrm{rpm}$ for approximately $5 \mathrm{~min}$. The samples settled for $15 \mathrm{~min}$, after which $0.5 \mathrm{ml}$ of supernatant from each sample was transferred to a $15-\mathrm{ml}$ centrifuge tube. To dilute the supernatant to a final 40 -fold dilution, $9.5 \mathrm{ml}$ of distilled water was added to each $15-\mathrm{ml}$ tube. FUM concentration was quantified with FUM-specific enzyme-linked immunosorbent assay (ELISA) kits (Helica Biosystems, Inc., Santa Ana, CA). Absorbance at $450 \mathrm{~nm}$ of the ELISA plates was read using a BioTek $\mu$ Quant microplate spectrophotometer (BioTek Instruments, Inc., Winooski, VT) paired with Gen5 software (BioTek Instruments, Inc.). Samples that had FUM predicted to be above the highest standard provided by the ELISA kits, $6 \mu \mathrm{g} \mathrm{g}^{-1}$ (ppm), were serially diluted until their predicted FUM was within the standard curve. Box-Cox-transformed FUM did not differ between duplicates (oneand two-tailed $t$ tests, $P>0.05$ ); as such, FUM was averaged over the two duplicates for further analyses. The ratio of FUM to FER (FUM:FER) was calculated as FUM/(FER + 1). One was added to all FER data to adjust $0 \%$ FER scores in the denominator.

BIK was extracted from ground bulk samples as described by Busman et al. (2012). A 0.2-g subsample from each ground bulk and $1 \mathrm{ml}$ of $1: 1$ acetonitrile/ethyl acetate were added to a 2-ml centrifuge tube, and the tubes were occasionally mixed for $3 \mathrm{~h}$. The supernatant

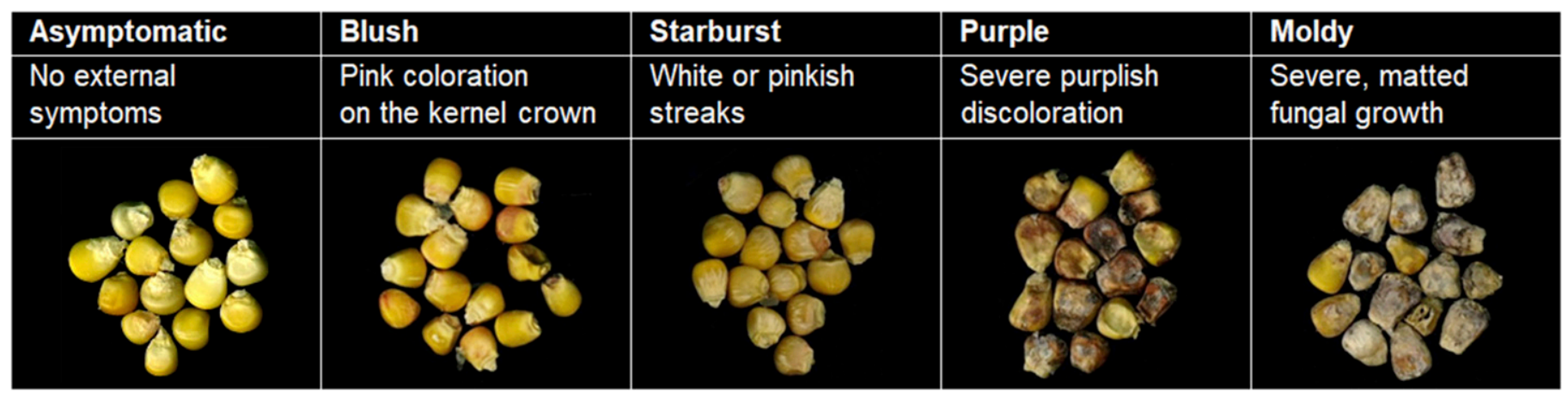

Fig. 1. Descriptions and photographic examples of Fusarium verticillioides-inoculated kernels exhibiting five distinct symptom types. 
from each sample was then transferred to a clean 2-ml centrifuge tube. Stock solution of BIK at $10 \mathrm{ppm}$ was prepared by dissolving pure BIK (AdipoGen Corp., San Diego, CA) in 1:1 acetonitrile/ ethyl acetate. Five other BIK standards were prepared by diluting the stock solution further with $1: 1$ acetonitrile/ethyl acetate to final concentrations of $0.25,0.5,1,2.5$, and $5 \mathrm{ppm}$. The six BIK standards (100 $\mu \mathrm{l} \mathrm{each)} \mathrm{and} 90$ unknown extracts were transferred to 96-well glass-coated microplates. Absorbance at $518 \mathrm{~nm}$ was read using a BioTek Synergy 2 multimode plate reader (BioTek Instruments, Inc.) paired with Gen5 software (BioTek Instruments, Inc.). To approximate samples that had nondetectable BIK levels $(<0.25 \mathrm{ppm})$, uniform random values between 0 and $0.25 \mathrm{ppm}$ were assigned to these samples (Lubin et al. 2004).

Seven maize lines (Supplementary Dataset S1) were selected for further analysis at the symptom type level based on two criteria: (i) the line was inoculated with both inoculation methods in the same plot in at least one field replication and (ii) the lines were representative of the spectrum of variation in subplot-level FUM, KBD, and BIK. Six lines had two paired subplot samples each: one point and one inundatively inoculated subplot sample from the same plot. One line had four paired subplot samples: two point and two inundatively inoculated subplot samples from two different plots. From each of the 16 total subplot samples, approximately 25 asymptomatic kernels and 25 symptomatic kernels exhibiting the same symptom type were randomly selected. This yielded 44 total "discrete symptom type" samples representative of the five symptom types described previously. KBD of each 25-kernel sample was calculated as the weight divided by the bulk volume of the 25 kernels. Approximately five kernels were randomly selected from each sample, weighed, and ground to a fine powder using an IKA Tube Mill (IKA Works, Inc., Wilmington, NC); FUM was then extracted and quantified using the same ELISA kit protocols described previously (Helica Biosystems, Inc.). Approximately 10 kernels were randomly selected from each sample and ground to a fine powder using an IKA Tube Mill (IKA Works, Inc.); BIK was then extracted and quantified using the protocol described previously.

Cob phenotyping. For each cob within each subplot, we measured its length (CobLen), diameter at the halfway point of its length (CobDiam) (Brown et al. 2011), and weight (CobMass).
Assuming cob shape to be cylindrical, cob volume (CobVol) was calculated as $\pi$ CobLen (CobDiam $/ 2)^{2}$, and cob density (CobDen) was calculated as CobMass/CobVol. The average CobLen, CobDiam, CobMass, CobVol, and CobDen were then calculated for each subplot.

Data transformation. The raw CobDen and disease phenotypes were not normally distributed. Box-Cox transformations were performed using JMP software (SAS Institute Inc., Cary, NC) to normalize the subplot data, where $\lambda=0$ for FUM, FUM:FER, and BIK; $\lambda=0.2$ for FER; $\lambda=2$ for KBD; and $\lambda=-1.4$ for CobDen. The raw FUM, KBD, and BIK data from discrete symptom type samples were also Box-Cox-transformed, where $\lambda=0,2$, and 0.4 , respectively.

Comparison of disease severity and cob morphology between inoculation methods. Unless noted otherwise, all analyses described hereafter were conducted using JMP software (SAS Institute Inc.). Box-Cox-transformed FER, FUM, FUM:FER, KBD, BIK, CobLen, CobDiam, CobMass, CobVol, and CobDen under the two inoculation methods were compared with one-tailed and two-tailed $t$ tests. To compare the composition of symptom types under the two inoculation methods, we used a $\chi^{2}$ test. We also fit general linear models (assuming a binomial distribution and employing a logit link function) with the absence or presence of each symptom type as the response and inoculation method as a predictor. Pearson correlations among Box-Cox-transformed FER, FUM, KBD, BIK, CobLen, CobDiam, CobMass, CobVol, and CobDen were estimated for all subplot data combined and by inoculation method.

Genetic variation for disease resistance and cob morphology. To assess genotypic, inoculation method, and field effects on disease severity and cob morphology, mixed linear models (MLM) were fit to include Box-Cox-transformed FER, FUM, FUM:FER, KBD, BIK, CobLen, CobDiam, CobMass, CobVol, and CobDen as separate response variables. MLM included (i) inoculation method, genotype, and their interaction (G-I) as fixed effects and field replication and block[replication] as random for all subplot data combined; and (ii) genotype as a fixed effect and replication and block[replication] as random for inoculation method-specific data.

To investigate genetic correlations among disease severity and cob morphology, multivariate MLM were fit with Box-Cox-transformed

TABLE 1. Comparison of Fusarium ear rot (FER), fumonisin (FUM) concentration, ratio of FUM to Fusarium ear rot (FUM:FER), kernel bulk density (KBD), and bikaverin (BIK) in subplots exhibiting distinct symptom types ${ }^{\mathrm{z}}$

\begin{tabular}{|c|c|c|c|c|c|c|}
\hline Statistic & $N$ & FER $(\%)$ & FUM (ppm) & FUM:FER (ppm \%-1) & $\mathrm{KBD}\left(\mathrm{g} \mathrm{ml}^{-1}\right)$ & BIK (ppm) \\
\hline Blush mean & 15 & $50.1 \pm 2.9 \mathrm{~A}$ & $30.3 \pm 3.6 \mathrm{~A}$ & $0.66 \pm 0.12 \mathrm{AB}$ & $0.689 \pm 0.015 \mathrm{~A}$ & $1.29 \pm 0.21 \mathrm{~A}$ \\
\hline Starburst mean & 34 & $45.5 \pm 2.6 \mathrm{~A}$ & $22.0 \pm 2.7 \mathrm{AB}$ & $0.43 \pm 0.08 \mathrm{~B}$ & $0.691 \pm 0.006 \mathrm{~A}$ & $1.40 \pm 0.11 \mathrm{~A}$ \\
\hline Purple mean & 79 & $38.5 \pm 1.2 \mathrm{~B}$ & $27.3 \pm 1.3 \mathrm{~A}$ & $0.85 \pm 0.05 \mathrm{~A}$ & $0.688 \pm 0.004 \mathrm{~A}$ & $1.47 \pm 0.06 \mathrm{~A}$ \\
\hline Moldy mean & 42 & $35.3 \pm 1.6 \mathrm{~B}$ & $21.6 \pm 2.7 \mathrm{~B}$ & $0.61 \pm 0.07 \mathrm{~B}$ & $0.697 \pm 0.005 \mathrm{~A}$ & $1.31 \pm 0.10 \mathrm{~A}$ \\
\hline ANOVA $P$ value & 170 & $<0.0001 * * *$ & $0.07^{\mathrm{ms}}$ & $0.006^{* *}$ & 0.7 & 0.5 \\
\hline
\end{tabular}

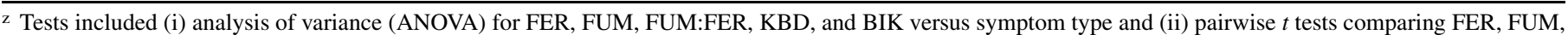
FUM:FER, KBD, or BIK between symptom types. FER, FUM, FUM:FER, KBD, and BIK were Box-Cox transformed before analysis. Transformed means and standard errors $( \pm)$ are reported in each symptom type/disease trait cell. Groups not connected by the same letter within each disease trait column are significantly different (pairwise two-tailed $t$ tests, $P<0.05$ ). Levels of significance of ANOVA: ${ }^{2}$ denotes $0.1>P \geq 0.05$, $* *$ denotes $0.01>P \geq 0.0001$, and $* * *$ denotes $P<$ 0.0001 .

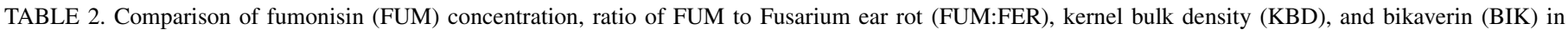
subplots exhibiting distinct symptom types after controlling for Fusarium ear rot (FER) severity ${ }^{\mathrm{z}}$

\begin{tabular}{|c|c|c|c|c|c|}
\hline Statistic & $N$ & FUM (ppm) & FUM:FER $\left(\mathrm{ppm} \%^{-1}\right)$ & $\mathrm{KBD}\left(\mathrm{g} \mathrm{ml}^{-1}\right)$ & BIK (ppm) \\
\hline Blush LS mean & 15 & $24.1 \pm 3.2 \mathrm{AB}$ & $0.63 \pm 0.17 \mathrm{AB}$ & $0.705 \pm 0.008 \mathrm{~A}$ & $1.20 \pm 0.16 \mathrm{~A}$ \\
\hline Starburst LS mean & 34 & $18.8 \pm 2.2 \mathrm{~B}$ & $0.40 \pm 0.11 \mathrm{~B}$ & $0.702 \pm 0.005 \mathrm{~A}$ & $1.34 \pm 0.11 \mathrm{~A}$ \\
\hline Purple LS mean & 79 & $28.6 \pm 1.4 \mathrm{~A}$ & $0.89 \pm 0.07 \mathrm{~A}$ & $0.685 \pm 0.003 \mathrm{~B}$ & $1.49 \pm 0.07 \mathrm{~A}$ \\
\hline Moldy LS mean & 42 & $23.8 \pm 2.0 \mathrm{~B}$ & $0.64 \pm 0.10 \mathrm{~B}$ & $0.689 \pm 0.005 \mathrm{AB}$ & $1.35 \pm 0.10 \mathrm{~A}$ \\
\hline Sym. type $P$ value & & $0.002 * *$ & $0.003 * *$ & $0.02 *$ & 0.3 \\
\hline FER $P$ value & & $<0.0001 * * *$ & 0.2 & $<0.0001 * * *$ & $0.02 *$ \\
\hline
\end{tabular}

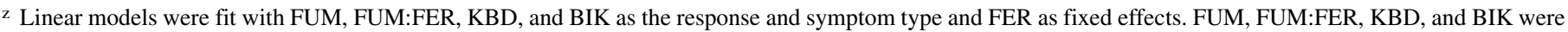
Box-Cox transformed before analysis. Transformed least-square (LS) means and standard errors ( \pm ) are reported in each symptom type/disease trait cell. Groups not connected by the same letter within each disease trait column are significantly different (pairwise two-tailed $t$ tests of LS means, $P<0.05$ ). Levels of significance for fixed effects: $*$ denotes $0.05>P \geq 0.01$, ** denotes $0.01>P \geq 0.0001$, and $* * * \operatorname{denotes} P<0.0001$. 
FER, FUM, FUM:FER, KBD, BIK, CobLen, CobDiam, CobMass, CobVol, and CobDen as the multivariate response with (i) inoculation method, genotype, and G-I as fixed effects and field replication and block[replication] as random for all subplot data combined; and (ii) genotype as a fixed effect and replication and block[replication] as random by inoculation method. Pearson correlations among the least-square means of the 10 disease and cob traits were estimated from the combined and inoculation method-specific multivariate models.

To estimate broad-sense heritability $(\mathrm{H})$, random linear models were fit with Box-Cox-transformed FER, FUM, FUM:FER, KBD, BIK, CobLen, CobDiam, CobMass, CobVol, and CobDen as separate response variables within and among inoculation methods. Genotype, replication, and block[replication] were modeled as random. H was estimated as $\sigma_{G}^{2} /\left(\sigma_{G}^{2}+\sigma_{e}^{2} / r\right)$, where $r$ is the number of field replications $(n=2)$, and $\sigma_{e}^{2}$ and $\sigma_{G}^{2}$ are the error and genetic variance components, respectively (Piepho and Moehring 2007).

Assessing potential proxies for FUM contamination. The following describe analyses conducted on data from discrete symptom type samples. Box-Cox-transformed FUM, KBD, and BIK versus symptom type were assessed with analysis of variance (ANOVA). Box-Cox-transformed FUM, KBD, and BIK between all pairs of symptom types were compared with one-tailed and twotailed $t$ tests. Box-Cox-transformed KBD and BIK of samples with raw FUM $<2$ ppm versus samples with FUM $\geq 2$ ppm were compared with one-tailed and two-tailed $t$ tests.

The KBD grand mean of all discrete symptom type samples combined (approximately $0.59 \mathrm{~g} \mathrm{ml}^{-1}$ ) was calculated and used as a threshold to separate samples into two groups: samples with $\mathrm{KBD}<0.59 \mathrm{~g} \mathrm{ml}^{-1}$ ("low-density") and samples with $\mathrm{KBD} \geq$ $0.59 \mathrm{~g} \mathrm{ml}^{-1}$ ("high-density"). The FUM content of each sample was calculated as the FUM concentration multiplied by the weight. The FUM content and weight of samples from the same subplot were added to calculate the total weight and FUM content of each subplot. Within each subplot, samples were assigned to low- and high-density groups and the FUM content and weight of each group were calculated. Percentages of FUM content and weight of the two density groups versus the total of the subplot were then calculated.

Linear discriminant analysis (LDA) was conducted using the MASS package (Venables and Ripley 2002) with fivefold crossvalidation replicated 10 times in $\mathrm{R}$ (version 3.3.1; $\mathrm{R}$ Foundation for Statistical Computing, Vienna). LDA models with Box-Coxtransformed KBD were constructed to discriminate discrete symptom type samples with FUM $<2$ ppm from samples with FUM $\geq 2$ ppm for all data combined and by inoculation method.

\section{RESULTS}

Discrete symptom types track FUM contamination and KBD. To dissect the relationships between symptomatology and FUM contamination and to identify low-cost proxies for FUM levels, we assessed disease severity with conventional and novel quantitative and qualitative methods. Of the quantitative phenotypes, FER and FUM contamination have been widely used as indicators of $F$. verticillioides infection severity (Bolduan et al. 2009; Parsons and Munkvold 2012; Presello et al. 2007; RobertsonHoyt et al. 2006; Zila et al. 2013). FER and FUM are phenotypically and genetically correlated (Robertson-Hoyt et al. 2007), and breeding programs have used FER for indirect selection of resistance to FUM because of the high costs associated with quantifying FUM (Horne et al. 2016). In addition, we used the ratio of FUM to FER (FUM: FER) as a measure of FUM load relative to the extent of visible infection. We measured KBD under the assumption that KBD would be an indicator of kernel tissue degradation by the fungus. BIK is a reddish pigment produced by $F$. verticillioides and other Fusarium spp., and its biosynthesis is coregulated with that of FUM (Lazzaro et al. 2012). We quantified BIK using a low-cost method modified from Busman et al. (2012), who demonstrated the use of BIK as an indicator of FER.

We devised a typology of FER symptoms and used it to describe the qualitative variation in external symptomatology (Fig. 1) and to relate visual symptoms to other disease traits. Only two subplots were asymptomatic and, thus, were excluded from all analyses

TABLE 3. Correlations among Fusarium ear rot (FER), fumonisin (FUM) concentration, ratio of FUM to Fusarium ear rot (FUM:FER), kernel bulk density (KBD), and bikaverin (BIK) among subplots exhibiting distinct symptom types ${ }^{2}$

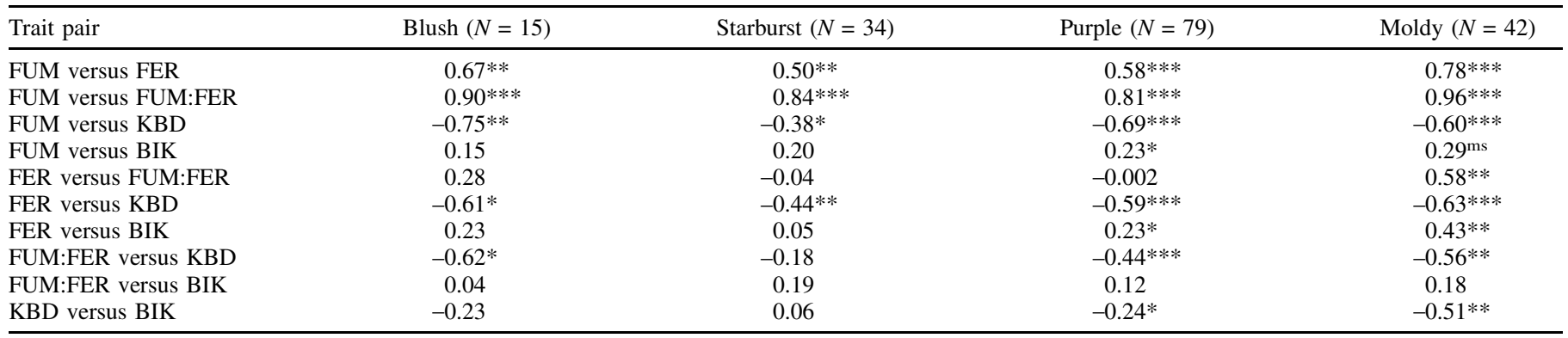

$\mathrm{z}$ The subplot dataset was split by symptom type. Pearson correlations were then calculated for all pairs of traits within each symptom type set. FER, FUM, FUM:

FER, KBD, and BIK were Box-Cox transformed before analysis. Pearson correlations are reported in each cell, with levels of significance as follows: ${ }^{2}$ denotes $0.1>P \geq 0.05$, * denotes $0.05>P \geq 0.01$, ** denotes $0.01>P \geq 0.0001$, and *** denotes $P<0.0001$.

TABLE 4. Comparison of kernel bulk density, fumonisin concentration, and bikaverin among discrete symptom type samples ${ }^{\mathrm{z}}$

\begin{tabular}{lrcrc}
\hline Statistic & $N$ & Bulk density $\left(\mathrm{g} \mathrm{m}^{-1}\right)$ & Fumonisins $(\mathrm{ppm})$ & Bikaverin $(\mathrm{ppm})$ \\
\hline Asymptomatic mean & 16 & $0.674 \pm 0.005 \mathrm{~A}$ & $0.111 \pm 0.009 \mathrm{C}$ & $4.556 \pm 0.430 \mathrm{AB}$ \\
Blush mean & 5 & $0.641 \pm 0.018 \mathrm{AB}$ & $8.726 \pm 7.404 \mathrm{~B}$ & $3.242 \pm 1.971 \mathrm{~B}$ \\
Starburst mean & 9 & $0.609 \pm 0.015 \mathrm{~B}$ & $2.657 \pm 1.288 \mathrm{~B}$ & $5.205 \pm 1.228 \mathrm{AB}$ \\
Purple mean & 7 & $0.477 \pm 0.024 \mathrm{C}$ & $349.282 \pm 85.209 \mathrm{~A}$ & $7.134 \pm 1.398 \mathrm{~A}$ \\
Moldy mean & 7 & $0.446 \pm 0.013 \mathrm{C}$ & $269.080 \pm 73.264 \mathrm{~A}$ & $5.549 \pm 1.165 \mathrm{AB}$ \\
ANOVA $P$ value & 44 & $<0.0001 * * *$ & $<0.0001 * * *$ & 0.2 \\
\hline
\end{tabular}

${ }^{\mathrm{z}}$ Each discrete symptom type sample was composed of approximately 25 kernels exhibiting the same symptom type. Raw means and standard errors ( \pm ) are reported in each symptom type/disease trait cell. Groups not connected by the same letter within each disease trait column are significantly different (pairwise two-tailed $t$ tests, $P<0.05$ ). Asterisks $(* * *)$ indicate $P<0.0001$ for analysis of variance (ANOVA) of disease trait in column header versus symptom type. ANOVA and pairwise $t$ tests were conducted on Box-Cox-transformed data. 
comparing quantitative disease traits (e.g., FUM and KBD) among symptom types at the subplot level. The multiple-symptom category was also excluded. We found that symptom type was significantly associated (ANOVA, $P<0.05$ ) with FER and FUM:FER and marginally significantly associated (ANOVA, $0.1>P \geq 0.05$ ) with FUM at the subplot level. Subplots exhibiting the purple and blush symptom types had greater FUM and FUM:FER than starburst and moldy subplots (Table 1); this was contrary to our hypothesis that subplots with extreme symptomatology (purple and moldy) would have greater quantitative disease severity than subplots with moderate symptoms (blush and starburst). However, blush and starburst subplots had greater FER scores than purple and moldy subplots (Table 1). To test whether these trends were conserved after controlling for FER, we fit linear models for FUM, FUM:FER, KBD, and BIK with symptom type and FER as fixed effects. After controlling for FER, we found similar differences in FUM, FUM: FER, KBD, and BIK among symptom types (Table 2). Both symptom type $(P<0.05)$ and FER $(P<0.0001)$ were significantly associated with FUM and KBD (Table 2). FUM:FER and BIK were significantly associated with symptom type $(P<0.01)$ and FER $(P<$ $0.05)$, respectively (Table 2 ). In the models where FER was significantly associated, FER had a positive effect on the response (FUM, KBD, and BIK) (Table 2).

Given these findings, we tested whether symptom type affected correlations among quantitative traits (e.g., FUM and KBD) at the subplot level. First, we split the subplot dataset by symptom type (all subplots exhibiting the blush symptom type in one set, all starburst subplots in another set, and so on) and then correlated all pairs of quantitative traits within each symptom type set. Subplots exhibiting multiple symptoms were excluded. We found a greater number of significant $(P<0.05)$ correlations between quantitative traits in purple and moldy subplots $(n=8$ significant correlations each) than in blush $(n=5)$ and starburst $(n=4)$ subplots (Table 3$)$; however, this trend may be due to the smaller number of blush $(n=$ $15)$ and starburst $(n=34)$ subplots than purple $(n=79)$ and moldy $(n=42)$ subplots. Correlations between FUM and FER and between FUM and FUM:FER were stronger in blush $(r=0.67$ and 0.90 , respectively) and moldy ( $r=0.78$ and 0.96 , respectively) subplots than in starburst ( $r=0.50$ and 0.84 , respectively) and purple
( $r=0.58$ and 0.81 , respectively) subplots (Table 3 ). Starburst plots yielded weaker KBD-FUM and KBD-FER correlations than the blush, purple, or moldy subplots (Table 3). Although FUM:FER is a ratio between and, in turn, is correlated with FUM and FER, correlations between these three phenotypes and other traits were not necessarily conserved across inoculation methods or symptom types. This suggests that FUM accumulation and visible symptom severity may be partially controlled by distinct mechanisms.

Within a given subplot, not all kernels were symptomatic or displayed the same symptoms. In light of this heterogeneity, we investigated relationships among indicators of external and internal infection severity at a finer scale. We pooled groups of kernels exhibiting the same symptom type and tested them for KBD, FUM, and BIK. In these discrete symptom type samples, we found that symptom type tracked FUM and KBD. Specifically, purple and moldy samples had the greatest FUM and the lowest KBD, while asymptomatic kernels had the least FUM and highest KBD (Table 4). The blush and starburst types were intermediate with respect to FUM and KBD (Table 4). BIK was not significantly associated with symptom type but pairwise $t$ tests revealed that purple kernels had higher BIK than blush kernels (Table 4).

TABLE 5. Prediction accuracy, specificity, sensitivity, and type I and II error rates for linear discriminant analysis (LDA) using kernel bulk density to predict fumonisin (FUM) status $(<2$ or $\geq 2 \mathrm{ppm})$ in discrete symptom type samples $^{\mathrm{z}}$

\begin{tabular}{lccccc}
\hline Analysis & $R^{2}$ & Sensitivity & Specificity & Type I error & Type II error \\
\hline Combined & 0.63 & 0.80 & 0.97 & 0.20 & 0.03 \\
Point & 0.72 & 0.84 & 0.99 & 0.16 & 0.01 \\
Inundative & 0.60 & 0.78 & 0.97 & 0.22 & 0.03 \\
\hline
\end{tabular}

${ }^{\mathrm{z}}$ Based on fumonisin-specific enzyme-linked immunosorbent assay results, discrete symptom type samples were categorized as having $<2 \mathrm{ppm}$ or $\geq 2 \mathrm{ppm}$ FUM concentration; this FUM status was used as the response in LDA. LDA models were fivefold cross-validated with 10 replications. $R^{2}=$ Prediction accuracy of LDA model. Sensitivity $=$ proportion of samples with FUM $\geq 2$ ppm predicted to have FUM $\geq 2$ ppm. Specificity = proportion of samples with FUM $<2$ ppm predicted to have FUM $<2$ ppm. Type I error = proportion of samples with FUM $<2$ ppm predicted to have FUM $\geq 2$ ppm. Type II error = proportion of samples with FUM $\geq 2 \mathrm{ppm}$ predicted to have FUM $<2 \mathrm{ppm}$.
A

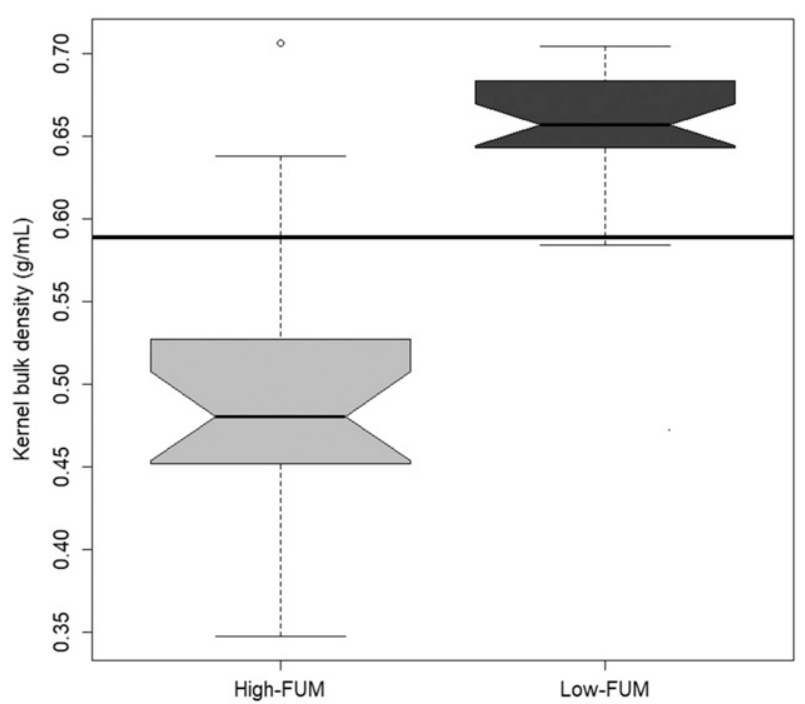

B

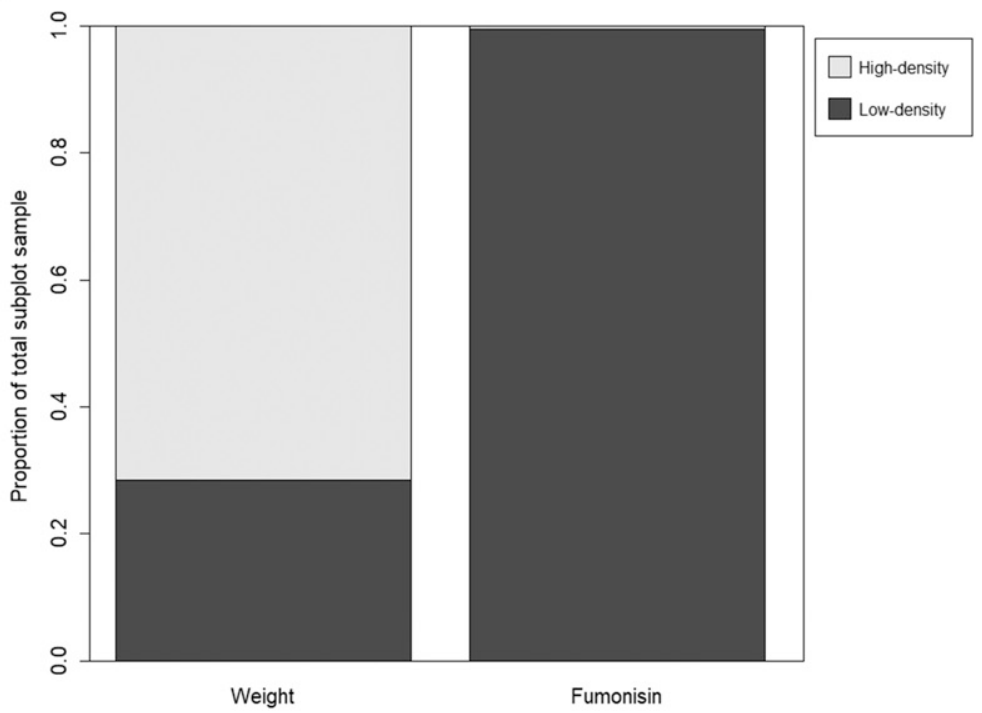

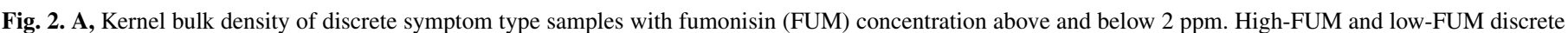

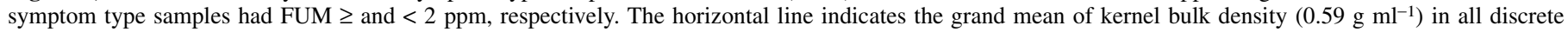

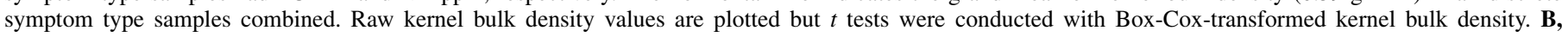

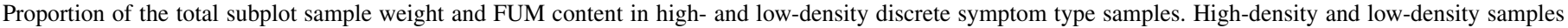
had kernel bulk densities $\geq$ and $<0.59 \mathrm{~g} \mathrm{ml}^{-1}$, respectively. 
KBD is predictive of FUM levels in discrete symptom type samples. FUM was highly positively correlated with KBD $\left(R^{2}=0.76, P<0.0001\right)$ but was not significantly correlated with $\operatorname{BIK}\left(R^{2}=0.06, P=0.12\right)$ in discrete symptom type samples. KBD was lower in discrete symptom type samples with FUM concentration above 2 ppm ("high-FUM"), the U.S. Food and Drug Administration guidance level, than in samples containing less than 2 ppm FUM ("low-FUM") (one- and two-tailed $t$ tests, $P<0.0001$ ) (Fig. 2A). BIK did not differ between low-FUM and high-FUM samples (one- and two-tailed $t$ tests, $P>0.05$ ).
Most of the toxin was associated with the lightest grain. A threshold based on the grand mean of KBD for all discrete symptom type samples $\left(0.59 \mathrm{~g} \mathrm{ml}^{-1}\right)$ was used to classify low-density $(\mathrm{KBD}<$ $\left.0.59 \mathrm{~g} \mathrm{ml}^{-1}\right)$ and high-density $\left(\mathrm{KBD} \geq 0.59 \mathrm{~g} \mathrm{ml}^{-1}\right)$ samples. Lowdensity samples represented a lower proportion of the total subplot sample weight (one- and two-tailed $t$ tests, $P<0.0001$ ) and a higher proportion of the total FUM content in the subplot sample (one- and two-tailed $t$ tests, $P<0.0001)$ than high-density samples from the same subplot sample (Fig. 2B). On average, low-density samples accounted for approximately one-quarter of the total subplot sample
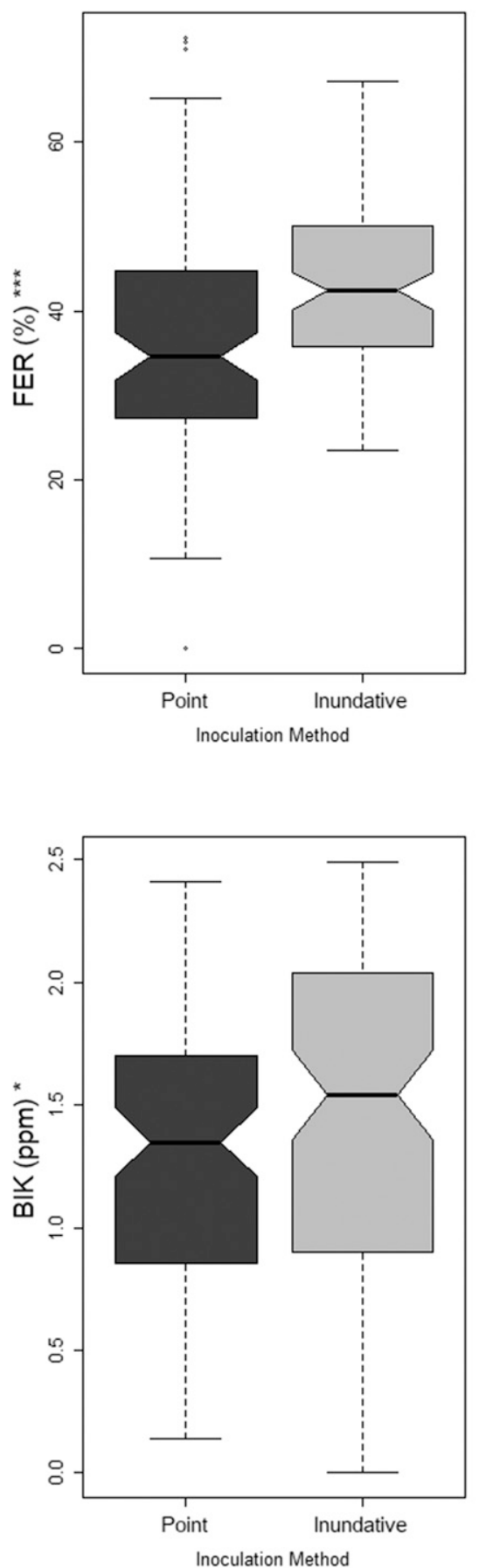
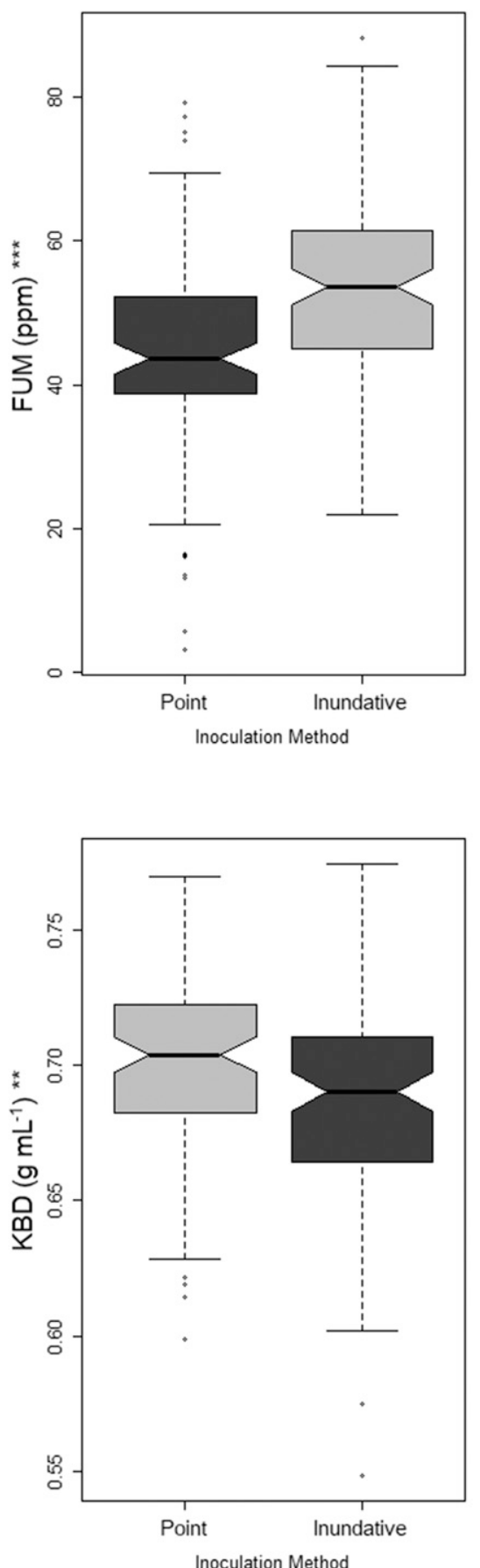
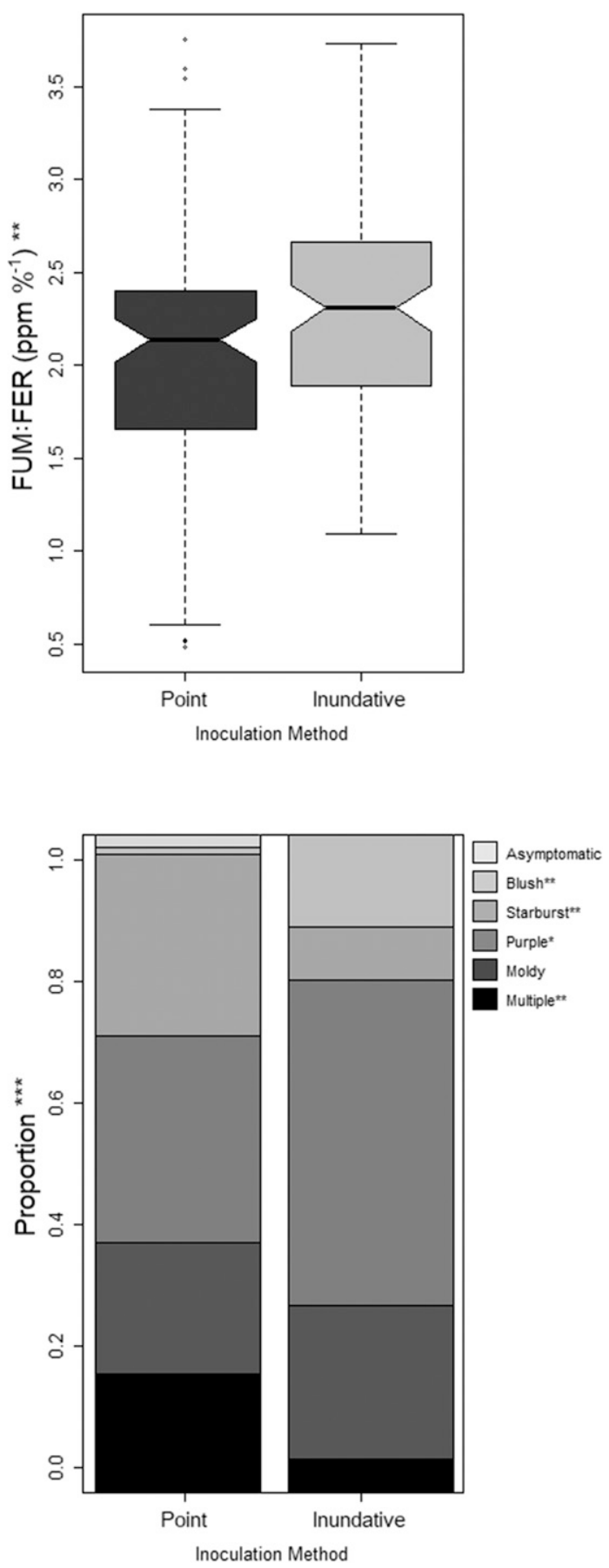

Fig. 3. Disease severity of subplots under point versus inundative inoculation. Fusarium ear rot (FER), fumonisin (FUM) concentration, ratio of FUM to Fusarium ear rot (FUM:FER), kernel bulk density (KBD), and bikaverin (BIK) were Box-Cox transformed before analysis. Transformed data are presented here. Two-tailed $t$ tests were used to compare FER, FUM, FUM:FER, KBD, and BIK between inoculation methods, and the significance of each test is denoted in the box plot y-axis titles. Proportions of subplots exhibiting the five symptom types under the two inoculation methods are presented as stacked bar plots. A $\chi^{2}$ test was used to compare symptom type composition between inoculation methods, and the significance of this test is denoted in the bar plot y-axis. Binomial models were used to compare the absence or presence of each symptom type between inoculation methods, and the significance of each test is denoted next to each symptom type in the bar plot legend. Significance for all tests described here: * denotes $0.05>P \geq 0.01$, ** denotes $0.01>P \geq 0.0001$, and $* * *$ denotes $P<0.0001$. 
weight but contained more than $99 \%$ of the total FUM content in the subplot sample (Fig. 2B).

Because KBD was highly correlated with FUM, we built LDA models using KBD to discriminate high-FUM and low-FUM discrete symptom type samples with fivefold cross-validation replicated 10 times. LDA had moderate accuracy to discriminate high-FUM and low-FUM samples, with higher accuracy for pointinoculated samples $\left(R^{2}=0.72\right)$ than for inundatively inoculated $\left(R^{2}=0.60\right)$ and combined samples $\left(R^{2}=0.63\right)$ (Table 5). The LDA models were able to detect high-FUM discrete symptom type samples 78 to $84 \%$ of the time (sensitivity) and low-FUM samples 97 to $99 \%$ of the time (specificity) (Table 5).

Disease severity is greater under inundative inoculation. Because inundative inoculation deposits a large volume of inoculum onto the ear, whereas point inoculation penetrates the ear at a single location, we expected to observe greater disease severity under inundative inoculation (Clements et al. 2003). Indeed, disease severity was greater under inundative than point inoculation, as demonstrated by both quantitative and qualitative phenotypes (Fig. 3 ). Compared with point-inoculated subplots, inundatively inoculated subplots had greater FER, FUM, and BIK and more greatly reduced KBD (Fig. 3). Notably, inundative inoculation also yielded greater toxin load per visibly infected area (FUM:FER) (Fig. 3).

Symptom typology differed significantly between the two inoculation methods $\left(\chi^{2}=38, P<0.0001\right)$, with a greater representation of severe and pigmented symptoms under inundative inoculation (Fig. 3). The inundative method yielded a greater proportion of purple and blush subplots and a smaller proportion of starburst subplots than point inoculation (Fig. 3). The point inoculation method led to a more uniform spread of symptom types and a larger number of subplots exhibiting multiple symptoms (Fig. 3). Only point inoculation yielded asymptomatic subplots $(n=2)$.

Inoculation method influences genetic variation for resistance to $\boldsymbol{F}$. verticillioides infection. FER and FUM were positively correlated with each other (phenotypically and genetically) and negatively correlated with KBD, regardless of inoculation method (Table 6). Across inoculation methods, the phenotypic correlation between FUM and KBD was stronger than that of FER and KBD (Table 6). This could have been partially due to our scoring of FER while the kernels were still attached to the ear, which may have hindered our observation of symptoms on the lower portion of the kernels. Not all correlations between disease phenotypes were conserved across inoculation methods (Table 6). Point inoculation resulted in a greater number of significant phenotypic correlations and a smaller number of significant genetic correlations than inundative inoculation (Table 6).

Given the differences in disease severity and disease phenotype correlations between inoculation methods, we sought to test whether the two inoculation methods revealed different genetic sources of resistance. This was not the case, as demonstrated by the significant association of inoculation method and genotype-but not their interaction - with FER, FUM, and KBD (Table 7). Both methods reliably ranked genotypes for resistance to FER and FUM. GE440, an inbred line previously reported as an excellent source of resistance (Clements et al. 2004; Robertson et al. 2006), was one of the most resistant genotypes across inoculation methods. Similar to previous findings (Clements et al. 2004; Robertson et al. 2006; Zila et al. 2013, 2014), FR1064 and B73 were among the most susceptible lines for FER and FUM under both inoculation methods. The relationship among FER, FUM, and KBD was further

TABLE 6. Phenotypic and genetic correlations among disease and cob traits within and among inoculation methods ${ }^{\mathrm{z}}$

\begin{tabular}{|c|c|c|c|c|c|c|c|c|c|c|}
\hline Method & FER & FUM & FUM:FER & BIK & KBD & Cob Den & Cob Diam & Cob Len & Cob Mass & Cob Vol \\
\hline \multicolumn{11}{|l|}{ Combined } \\
\hline FER & $\ldots$ & $0.59 * * *$ & 0.08 & $0.16^{*}$ & $-0.49 * * *$ & $0.12^{\mathrm{ms}}$ & 0.06 & $0.14 *$ & $0.16^{*}$ & 0.07 \\
\hline FUM & $0.71 * * *$ & $\ldots$ & $0.84 * * *$ & $0.22 * *$ & $-0.51 * * *$ & $0.14 *$ & 0.09 & 0.05 & $0.16^{*}$ & 0.06 \\
\hline FUM:FER & $0.28 *$ & $0.88 * * *$ & $\ldots$ & $0.17^{*}$ & $-0.33 * * *$ & 0.11 & 0.06 & -0.04 & 0.1 & 0.02 \\
\hline BIK & $0.28^{\mathrm{ms}}$ & $0.32 *$ & $0.25^{\mathrm{ms}}$ & $\ldots$ & $-0.21 * *$ & 0.06 & 0.01 & -0.06 & 0.02 & -0.03 \\
\hline KBD & $-0.49 * *$ & $-0.61 * * *$ & $-0.49 * *$ & $-0.28^{*}$ & $\ldots$ & 0.08 & $-0.19 * *$ & -0.03 & -0.1 & $0.14^{\mathrm{ms}}$ \\
\hline Cob Den & $0.26^{\mathrm{ms}}$ & 0.23 & 0.15 & 0.06 & 0.12 & $\ldots$ & $-0.44 * * *$ & -0.05 & $0.19 * *$ & $-0.29 * * *$ \\
\hline Cob Diam & 0.05 & 0.10 & 0.08 & 0.11 & $-0.31 *$ & $-0.42 * *$ & $\ldots$ & $0.49 * * *$ & $0.69 * * *$ & $0.9 * * *$ \\
\hline Cob Len & $0.33 *$ & 0.11 & -0.09 & -0.05 & -0.14 & -0.05 & $0.51 * *$ & $\ldots$ & $0.79 * * *$ & $0.79 * * *$ \\
\hline Cob Mass & $0.30 *$ & 0.23 & 0.10 & 0.07 & -0.23 & 0.18 & $0.73^{* * *} *$ & $0.80 * * *$ & $\ldots$ & $0.86^{* * * *}$ \\
\hline Cob Vol & 0.12 & 0.08 & 0.01 & 0.03 & $-0.26^{\mathrm{ms}}$ & $-0.28 *$ & $0.91 * * *$ & $0.79 * * *$ & $0.88 * * *$ & $\ldots$ \\
\hline \multicolumn{11}{|l|}{ Point } \\
\hline FER & $\ldots$ & $0.68 * * *$ & $0.27 *$ & $0.28 *$ & $-0.44 * * *$ & $0.27 *$ & 0.07 & $0.26^{*}$ & $0.32 * *$ & 0.14 \\
\hline FUM & $0.51 * *$ & $\ldots$ & $0.89 * * *$ & $0.39 * *$ & $-0.57 * * *$ & $0.28^{*}$ & 0.09 & 0.08 & $0.26^{*}$ & 0.09 \\
\hline FUM:FER & -0.09 & $0.81 * * *$ & $\ldots$ & $0.33 * *$ & $-0.48 * * *$ & $0.19^{\mathrm{ms}}$ & 0.08 & -0.07 & 0.14 & 0.03 \\
\hline BIK & 0.18 & 0.19 & 0.11 & $\ldots$ & $-0.26^{*}$ & $0.19^{\mathrm{ms}}$ & 0.16 & -0.08 & 0.09 & 0.07 \\
\hline KBD & $-0.39 * *$ & $-0.38 * *$ & -0.17 & -0.08 & $\ldots$ & 0.14 & $-0.36^{* *}$ & -0.03 & $-0.23^{*}$ & -0.27 \\
\hline Cob Den & 0.10 & 0.23 & 0.21 & 0.19 & $0.28^{\mathrm{ms}}$ & $\ldots$ & $-0.44 * * *$ & -0.03 & $0.19^{\mathrm{ms}}$ & $-0.29 *$ \\
\hline Cob Diam & 0.02 & 0.05 & 0.02 & 0.04 & $-0.35^{*}$ & $-0.39 * *$ & $\ldots$ & $0.46 * * *$ & $0.70 * * *$ & $0.91 * * *$ \\
\hline Cob Len & $0.39 * *$ & 0.14 & -0.13 & 0.09 & -0.18 & 0.04 & $0.05^{* *}$ & $\ldots$ & $0.79 * * *$ & $0.77 * * *$ \\
\hline Cob Mass & $0.24^{\mathrm{ms}}$ & 0.21 & 0.05 & 0.18 & -0.19 & $0.28^{\mathrm{ms}}$ & $0.69 * * *$ & $0.80 * * *$ & $\ldots$ & $0.87 * * *$ \\
\hline Cob Vol & 0.14 & 0.07 & -0.04 & 0.07 & $-0.31^{*}$ & -0.22 & $0.91 * * *$ & $0.79 * * *$ & $0.86^{* * *}$ & $\ldots$ \\
\hline \multicolumn{11}{|l|}{ Inundative } \\
\hline FER & $\ldots$ & $0.63 * * *$ & 0.07 & 0.18 & $-0.58 * * *$ & 0.17 & 0.05 & $0.24 *$ & 0.15 & 0.04 \\
\hline FUM & $0.67 * * *$ & $\ldots$ & $0.82 * * *$ & 0.20 & $-0.63^{* * *} *$ & 0.14 & 0.07 & 0.07 & 0.12 & 0.02 \\
\hline FUM:FER & $0.30 *$ & $0.91 * * *$ & $\ldots$ & 0.13 & $-0.39 * *$ & 0.08 & 0.05 & -0.11 & 0.02 & -0.03 \\
\hline BIK & 0.16 & $0.35^{*}$ & $0.36^{* *}$ & $\ldots$ & $-0.26^{*}$ & 0.15 & -0.11 & -0.14 & -0.08 & -0.08 \\
\hline KBD & $-0.57 * * *$ & $-0.70 * * *$ & $-0.59 * * *$ & $-0.34^{*}$ & $\ldots$ & -0.05 & -0.14 & -0.17 & -0.14 & -0.14 \\
\hline Cob Den & $0.34^{*}$ & 0.23 & 0.10 & -0.06 & -0.08 & $\ldots$ & $-0.41 * *$ & -0.05 & 0.16 & $-0.28 *$ \\
\hline Cob Diam & 0.08 & 0.12 & 0.11 & 0.05 & -0.19 & $-0.46^{* *}$ & $\ldots$ & $0.55 * * *$ & $0.75 * * *$ & $0.92 * * *$ \\
\hline Cob Len & 0.15 & 0.07 & 0.04 & -0.15 & -0.03 & -0.13 & $0.50 * *$ & $\ldots$ & $0.82 * * *$ & $0.81 * * *$ \\
\hline Cob Mass & $0.28^{\mathrm{ms}}$ & $0.25^{\mathrm{ms}}$ & 0.16 & -0.06 & -0.20 & 0.09 & $0.75^{* * *}$ & $0.78 * * *$ & $\ldots$ & $0.89 * * *$ \\
\hline Cob Vol & 0.08 & 0.09 & 0.07 & -0.05 & -0.14 & $-0.34 *$ & $0.91 * * *$ & $0.78 * * *$ & $0.89 * * *$ & $\ldots$ \\
\hline
\end{tabular}

z Phenotypic correlations are in the upper diagonals. Genetic correlations among multivariate least-square means after controlling for genetic and field effects are in the lower diagonals. FER $=$ Fusarium ear rot, FUM = fumonisin concentration, FUM:FER = ratio of FUM to Fusarium ear rot, BIK = bikaverin, KBD = kernel bulk density, CobDen = cob density, CobDiam = cob diameter, CobLen = cob length, CobMass = cob mass, and CobVol = cob volume. FER, FUM, FUM:FER, BIK, KBD, and CobDen were Box-Cox transformed before analysis. Pearson correlations are displayed in each cell, with significance as follows: ${ }^{2}$ denotes 0.1 $>P \geq 0.05$ (marginally significant), * denotes $0.05>P \geq 0.01$, ** denotes $0.01>P \geq 0.0001$, and *** denotes $P<0.0001$. 
demonstrated by the fact that the most resistant and the most susceptible (with respect to FER and FUM) genotypes were also among the lines with the highest and lowest KBD, respectively. The interaction between genotype and inoculation method was marginally associated $(P<0.1)$ with FUM:FER (Table 7$)$.

Inundative inoculation may be more effective for screening genetic sources of resistance, as demonstrated by the higher broadsense $\mathrm{H}$ for FER, FUM, and KBD under inundative inoculation $(0.81$ to 0.85$)$ than in the combined $(0.45$ to 0.69$)$ and point $(0.51$ to 0.75 ) analyses (Table 8). H for FUM:FER was comparable under the two inoculation methods (0.75) (Table 8). Only point inoculation revealed genetic variation for BIK (Tables 7 and 8), indicating that differential BIK production may only be stimulated under point inoculation.

Cob morphology is an important component of resistance under point inoculation. Cob morphology did not differ between the two inoculation methods (one- and two-tailed $t$ tests $P>0.05$ for all cob phenotypes). Phenotypic and genetic correlations among cob traits were not influenced by inoculation method (Table 6). As expected (Jansen et al. 2015), we found that cob size (CobLen, CobDiam, and CobVol) traits were positively correlated with each other $(r=0.46$ to $0.92, P<0.05)$ and with CobMass ( $r=0.69$ to $0.89, P<0.05)$, and that CobDen was positively correlated with CobMass $(r=0.19$ to $0.28, P<0.05)$ and negatively correlated with cob size traits $(r=-0.19$ to $0.44, P<0.05)$ (Table 6).

In general, cob size was positively correlated with disease severity (Table 6). Some of the phenotypic and genetic correlations between disease severity and cob traits were shared between inoculation methods (Table 6). Across all analyses (combined and inoculation-method specific), FER and FUM were positively phenotypically or genetically correlated with cob traits. Specifically, FER was positively correlated with CobDen $(r=0.12$ to 0.34 , $P<0.05)$, CobLen $(r=0.14$ to $0.39, P<0.05)$, and CobMass $(r=$ 0.16 to $0.32, P<0.05)$, and FUM was positively correlated with CobMass ( $r=0.16$ to $0.26, P<0.05$ ) (Table 6 ). These positive correlations between CobDen and disease severity were contrary to our assumption that CobDen would be an indicator of cob tissue hardness, which could serve as a physical barrier against $F$. verticillioides infection. However, these CobDen-disease correlations were largely driven by CobMass; in linear models including both CobDen and CobMass with FER, FUM, or KBD as the response, only CobMass was significantly $(P<0.05)$ associated.

Cob morphology appeared to play a larger role in resistance to $F$. verticillioides infection under point inoculation. A larger number of disease-cob trait pairs (e.g., FUM and CobDen or FER and CobLen) were significantly $(P<0.05)$ or marginally significantly $(P<0.1)$ phenotypically or genetically correlated under point inoculation $(n=10)$ than under inundative inoculation $(n=4)$ and in the combined analysis $(n=7)$. In the point-specific and combined analyses, FUM was positively phenotypically correlated with CobDen $(r=0.14$ to $0.28, P<0.05)$, and $\mathrm{KBD}$ was negatively (phenotypically and genetically) correlated with CobDiam $(r=$ -0.19 to $0.36, P<0.01)$ and $\mathrm{CobVol}(r=-0.14$ to $0.31, P<0.1)$ (Table 6). Point inoculation revealed three unique disease-cob correlations: (i) FUM:FER was positively phenotypically correlated with CobDen $(r=0.19, P<0.1)$, (ii) KBD was negatively phenotypically correlated with CobMass $(r=0.23, P<0.05)$, and (iii) KBD was positively genetically correlated with CobDen

TABLE 7. Inoculation (Inoc) method, genotype, genotype-inoculation method (G-I), and field effects on disease and cob phenotypes ${ }^{\mathrm{z}}$

\begin{tabular}{|c|c|c|c|c|c|c|c|}
\hline \multirow[b]{2}{*}{ Analysis } & \multirow[b]{2}{*}{ Response } & \multicolumn{3}{|c|}{ Fixed effects ( $P$ values) } & \multicolumn{3}{|c|}{ Random effects (variance proportions) } \\
\hline & & Genotype & Inoc method & G-I & Rep & Block[Rep] & Error \\
\hline \multirow[t]{7}{*}{ Combined } & FUM & $<0.0001 * * *$ & $<0.0001 * * *$ & 0.72 & 0 & 3.4 & 96.6 \\
\hline & FUM:FER & $<0.0001 * * *$ & $0.0003 * *$ & $0.06^{\mathrm{ms}}$ & 0 & 6.2 & 93.8 \\
\hline & KBD & $<0.0001 * * *$ & $<0.0001 * * *$ & 0.75 & 0 & 0 & 100 \\
\hline & BIK & 0.85 & $0.03 *$ & 0.14 & 2.9 & 11.0 & 86.1 \\
\hline & CobDen & $<0.0001 * * *$ & 0.80 & 0.90 & 1.1 & 7.1 & 91.8 \\
\hline & CobMass & $<0.0001 * * *$ & $0.02 *$ & $0.05^{\mathrm{ms}}$ & 0 & 15.5 & 84.5 \\
\hline & CobVol & $<0.0001 * * *$ & 0.11 & 0.28 & 0 & 4.9 & 95.1 \\
\hline \multirow[t]{5}{*}{ Point } & FUM & $0.01 *$ & $\ldots$ & $\ldots$ & 0 & 1.4 & 98.6 \\
\hline & FUM:FER & $0.02 *$ & $\ldots$ & $\ldots$ & 1.4 & 26.0 & 72.6 \\
\hline & FER & 0.12 & $\ldots$ & $\ldots$ & 8.1 & 21.9 & 70.0 \\
\hline & KBD & $0.01 *$ & $\ldots$ & $\ldots$ & 0 & 0 & 100 \\
\hline & BIK & $0.09^{\mathrm{ms}}$ & $\ldots$ & $\ldots$ & 5.9 & 10.7 & 83.4 \\
\hline \multirow[t]{10}{*}{ Inundative } & FUM & $<0.0001 * * *$ & $\ldots$ & $\ldots$ & 0 & 14.0 & 86.0 \\
\hline & FUM:FER & $0.0002 * *$ & $\ldots$ & $\ldots$ & 0 & 6.1 & 93.9 \\
\hline & FER & $0.0006 * *$ & $\ldots$ & $\ldots$ & 10.1 & 0.3 & 89.6 \\
\hline & KBD & $<0.0001 * * *$ & $\ldots$ & $\ldots$ & 0 & 0 & 100 \\
\hline & BIK & 0.63 & $\ldots$ & $\ldots$ & 0 & 24.7 & 75.3 \\
\hline & CobDen & $<0.0001 * * *$ & $\ldots$ & $\ldots$ & 0 & 11.2 & 88.8 \\
\hline & CobDiam & $<0.0001 * * *$ & $\ldots$ & $\ldots$ & 0 & 27.6 & 72.4 \\
\hline & CobLen & $<0.0001 * * *$ & $\ldots$ & $\ldots$ & 0 & 0 & 100 \\
\hline & CobMass & $<0.0001 * * *$ & $\ldots$ & $\ldots$ & 0.1 & 4.3 & 95.6 \\
\hline & CobVol & $<0.0001 * * *$ & $\ldots$ & $\ldots$ & 0 & 21.7 & 78.3 \\
\hline
\end{tabular}

z Linear models were fit with (i) genotype, inoculation method, and G-I as fixed effects and field replication and block[replication] (Block[Rep]) as random effects for the combined analyses, and (ii) genotype as a fixed effect and field replication and block[replication] as random effects for the inoculation method-specific analyses. FER = Fusarium ear rot, FUM = fumonisin concentration, FUM:FER = ratio of FUM to Fusarium ear rot, BIK = bikaverin, KBD = kernel bulk density, CobDen $=$ cob density, CobDiam $=$ cob diameter, CobLen $=$ cob length, CobMass = cob mass, and CobVol $=$ cob volume. FER, FUM, FUM:FER, KBD, BIK, and CobDen were Box-Cox transformed before analysis. Fixed effect $P$ value significance as follows: ms denotes $P<0.1$ (marginally significant), ${ }^{*}$ denotes $0.05>P \geq 0.01, * *$ denotes $0.01>P \geq 0.0001$, and $* *$ denotes $P<0.0001$. 
$(r=0.28, P<0.1)$ (Table 6). No disease-cob correlations were unique to inundative inoculation (Table 6).

\section{DISCUSSION}

In this study, we identified potential proxies for FUM contamination and found that inoculation methods representing distinct infection pathways revealed different relationships between cob and kernel morphology and resistance to $F$. verticillioides in maize. To our knowledge, we are the first to report the association of cob morphology with mycotoxin contamination and to explicitly demonstrate the use of KBD as an indicator of FUM status. The wide phenotypic variation resulting from combining the two inoculation methods allowed for a more detailed dissection of symptomatology than previous studies.

Our characterization of symptom typology complements published findings wherein symptomatic kernels had higher FUM contamination than asymptomatic kernels (Desjardins and Plattner 1998) and wherein visibly moldy kernels had greater FUM than starburst kernels (Parsons and Munkvold 2010, 2012) by further dividing external symptomatology into two moderate and two extreme types. We utilized this symptom typology to identify proxies for FUM contamination, which is expensive and timeconsuming to quantify. In our study, KBD was an accurate predictor of FUM content, indicating its potential use in grain sorting and breeding for FUM reduction. Variation in KBD may be directly due to kernel tissue degradation by $F$. verticillioides, innate grain characteristics (Fox and Manley 2009), or agronomic factors (Blandino et al. 2008; Miller 2001). Previous studies have demonstrated that traits related to kernel density such as hardness, increased grain weight, and reduced buoyancy were negatively associated with FER severity and FUM contamination under $F$. verticillioides infection (Blandino and Reyneri 2008; Presello et al. 2007, 2008; Shetty and Bhat 1999). It has generally been accepted in the industry that grain with lower $\mathrm{KBD}$ (also known as "test weight") is at greater risk for mycotoxin contamination (Patience and Ensley 2010; USDA-FGIS 2016). In addition, high phenotypic and genetic correlations among KBD, FUM, and FER indicate that resistance to FER and FUM contamination could potentially be accomplished via indirect selection of KBD under $F$. verticillioides inoculation. Similarly, Horne et al. (2016) successfully increased resistance to FUM accumulation via indirect selection for reduced FER.

We also tested BIK, a reddish pigment produced by $F$. verticillioides, as a proxy for FUM. Although BIK could not discriminate samples based on FUM status (using a threshold of $2 \mathrm{ppm}$ ), it was positively phenotypically and genetically correlated with FUM levels and FER, suggesting that it could be useful in breeding. The purple symptom type had the greatest concentration of BIK, which may be the causal pigment of the purple discoloration. In contrast, the localized pink coloration on the kernel crown characteristic of the blush type may explain the low BIK content of this symptom class. Unexpectedly, asymptomatic kernels, which had the lowest FUM contamination, had intermediate levels of BIK. Desjardins and Plattner (1998) found that, although asymptomatic kernels had lower FUM contamination than symptomatic kernels, F. verticillioides colonization was similar and extensive in both classes of kernels. In our study, F. verticillioides may have infected the asymptomatic kernels in our study and subsequently produced BIK in the internal, nonvisible kernel tissues.

Boutigny et al. (2012) previously showed a strong correlation $\left(R^{2}=0.98\right)$ between FUM concentration and the infection coefficient of $F$. verticillioides (ratio of $F$. verticillioides DNA mass to maize DNA mass, as determined by real-time polymerase chain reaction). However, we did not investigate the infection coefficient as a proxy for FUM contamination because it is not a low-cost method. Likewise, optical sorting has also showed promising results for detecting highly contaminated kernels in bulk grain samples (Dowell et al. 2002; Pearson et al. 2004, 2009; Stasiewicz et al.
2017) but we did not use these methods because they require specialized equipment.

Our results demonstrated that point and inundative inoculation methods reflected different modes of pathogenesis or resistance mechanisms. We found that cob morphology played a more important role in resistance under point than under inundative inoculation. Zummo and Scott (1990) demonstrated that natural F. verticillioides infection was greatest in the cob sclerenchyma and placenta tissues and that kernels were most frequently infected through the pedicel. The preference of the fungus to infect the pedicel over the other kernel tissues may be due to the combination of the low oxygen environment of the endosperm (Rolletschek et al. 2005) and the rich starch reserves in the lower pericarp adjacent to the pedicel (Bihmidine et al. 2013). Here, the large proportion of pointinoculated subplots exhibiting starburst symptoms - characterized by streaks radiating from the pedicel or the stylar canal (Costa et al. 2003; Duncan and Howard 2010; Parsons and Munkvold 2010, 2012; Presello et al. 2008)—suggests that the kernels may have been infected via the pedicel to some extent. Starburst streaking has been shown to be the result of fungal dissolution of pericarp cell walls and intracellular hyphal extension (Duncan and Howard 2010). Insect damage to the ear is an important avenue for $F$. verticillioides infection and it has been shown to be the major contributor to FER severity and FUM contamination in previous studies (Farrar and Davis 1991; Munkvold et al. 1997; Parsons and Munkvold 2010). Insect damage often provides an avenue for fungal entry into the cob; thus, it would be of interest to employ point inoculation methods to identify sources of resistance that may reduce the spread of the pathogen via the cob.

TABLE 8. Genotypic variance, field effect variance, and broad-sense heritability $(\mathrm{H})$ for disease and cob phenotypes ${ }^{\mathrm{z}}$

\begin{tabular}{|c|c|c|c|c|c|c|}
\hline \multirow[b]{2}{*}{ Analysis } & \multirow[b]{2}{*}{ Response } & \multicolumn{4}{|c|}{ Random effects (variance proportions) } & \multirow[b]{2}{*}{$\mathrm{H}$} \\
\hline & & Genotype & Rep & Block[Rep] & Error & \\
\hline \multirow[t]{10}{*}{ Combined } & FUM & 44.6 & 0 & 0 & 55.4 & 0.62 \\
\hline & FUM:FER & 32.5 & 0 & 0 & 67.5 & 0.49 \\
\hline & FER & 27.5 & 0 & 6.4 & 66.1 & 0.45 \\
\hline & KBD & 52.2 & 0 & 0 & 47.8 & 0.69 \\
\hline & BIK & 0 & 2.8 & 0 & 97.2 & 0 \\
\hline & CobDen & 54.8 & 0.7 & 2.2 & 42.3 & 0.72 \\
\hline & CobDiam & 79.0 & 0 & 0 & 21.0 & 0.88 \\
\hline & CobLen & 78.3 & 0 & 0 & 21.7 & 0.88 \\
\hline & CobMass & 83.1 & 0 & 0.7 & 16.2 & 0.91 \\
\hline & CobVol & 82.0 & 0 & 0.3 & 17.7 & 0.9 \\
\hline \multirow[t]{10}{*}{ Point } & FUM & 34.6 & 0 & 0 & 65.4 & 0.51 \\
\hline & FUM:FER & 34.8 & 2.5 & 2.4 & 60.3 & 0.54 \\
\hline & FER & 22.1 & 4.7 & 15.3 & 57.9 & 0.43 \\
\hline & KBD & 34.3 & 0 & 0 & 65.7 & 0.51 \\
\hline & BIK & 28.4 & 4.5 & 4.1 & 63.0 & 0.47 \\
\hline & CobDen & 29.5 & 3.4 & 0 & 67.1 & 0.47 \\
\hline & CobDiam & 66.4 & 0 & 0.2 & 33.4 & 0.80 \\
\hline & CobLen & 77.4 & 0 & 0 & 22.6 & 0.87 \\
\hline & CobMass & 83.9 & 0 & 0 & 16.1 & 0.91 \\
\hline & CobVol & 78.1 & 0.1 & 0.6 & 21.2 & 0.88 \\
\hline \multirow[t]{10}{*}{ Inundative } & FUM & 71.6 & 0 & 4.7 & 23.7 & 0.86 \\
\hline & FUM:FER & 58.8 & 0 & 2.9 & 38.3 & 0.75 \\
\hline & FER & 62.2 & 3.9 & 4.0 & 29.9 & 0.81 \\
\hline & KBD & 68.4 & 0 & 0 & 31.6 & 0.81 \\
\hline & BIK & 0 & 0.8 & 0.1 & 99.1 & 0 \\
\hline & CobDen & 67.3 & 0 & 2.0 & 30.7 & 0.81 \\
\hline & CobDiam & 86.4 & 0 & 2.4 & 11.2 & 0.94 \\
\hline & CobLen & 80.9 & 0 & 0 & 19.1 & 0.89 \\
\hline & CobMass & 86.3 & 0.1 & 0 & 13.6 & 0.93 \\
\hline & CobVol & 87.4 & 0 & 0.8 & 11.8 & 0.94 \\
\hline
\end{tabular}

z Linear models were fit with genotype, field replication, and block[replication] (Block[Rep]) as random effects. FER = Fusarium ear rot, FUM = fumonisin concentration, FUM:FER = ratio of FUM to Fusarium ear rot, BIK = bikaverin, $\mathrm{KBD}=$ kernel bulk density, CobDen $=$ cob density, CobDiam $=$ cob diameter, CobLen = cob length, CobMass = cob mass, and CobVol = cob volume. FER, FUM, FUM:FER, KBD, BIK, and CobDen were Box-Cox transformed before analysis. 
The larger, more widespread inoculum load of the inundative method likely allows more rapid and extensive fungal colonization than point inoculation, thereby implicating different modes of host defense or pathogenesis. This hypothesis is supported by the larger proportion of subplots exhibiting the severe purple symptom typology, greater FUM load per visibly infected area, and stronger correlations among disease traits observed under inundative than point inoculation. Likewise, Parsons and Munkvold (2012) found that FUM contamination was more highly correlated with the proportion of symptomatic kernels when the sample exhibited severe symptomatology (similar to our purple and moldy symptom types) than when the sample showed less severe, starburst symptoms. KBD was both genetically and phenotypically correlated with all four indicators of $F$. verticillioides infection severity and FUM contamination under inundative inoculation but with only two of these traits (FER and FUM) under point inoculation. This suggests that defense mechanisms related to the kernel tissues may be more relevant under inundative inoculation. In addition to the stronger correlations among disease traits, inundative inoculation yielded a smaller proportion of subplots exhibiting multiple symptoms than the point method, which may indicate that the manifestation of symptoms is more stable under inundative inoculation.

Across inoculation methods, we found that cob size traits were positively associated with disease severity and FUM contamination. Larger cobs may have more or larger vascular bundles, which could facilitate infection across the ear. Relative cob size phenotypes such as length and diameter are determined during inflorescence meristem development (Abendroth et al. 2011; Bommert et al. 2013). As such, mature cob size measurements should be unaffected by inoculation and should be indicative of immature cob morphology at the time of inoculation or natural infection by $F$. verticillioides (Abendroth et al. 2011; Bommert et al. 2013; Robertson-Hoyt et al. 2006; Zila et al. 2013), which occurs more than a month after inflorescence meristem development. In contrast, cob mass and density may be influenced by $F$. verticillioides infection. Because cob size is a component of grain yield (Jansen et al. 2015), the primary target of selection globally (Lorenz et al. 2010), trade-offs between yield and mycotoxin contamination should be assessed in the future.

We have demonstrated the importance of the mode of $F$. verticillioides infection on symptomatology and inference on resistance mechanisms in the maize ear. To maximize the effectiveness of selection on resistance to $F$. verticillioides and FUM contamination, we recommend that breeding trials utilize complementary inoculation methods that reflect diverse modes of infection and consider trade-offs between resistance and yield potential. In addition, KBD should be further examined as a proxy for FUM contamination in maize grain.

\section{ACKNOWLEDGMENTS}

We thank J. Brewer and J. Kolkman for technical support, J. Algarin for laboratory assistance, and N. Morales for data management.

\section{LITERATURE CITED}

Abendroth, L., Elmore, R., Boyer, M., and Marlay, S. 2011. Corn Growth and Development. Iowa State University, Ames.

Afolabi, C. G., Bandyopadhyay, R., Leslie, J. F., and Ekpo, E. J. A. 2006. Effect of sorting on incidence and occurrence of fumonisins and Fusarium verticillioides on maize from Nigeria. J. Food Prot. 69:2019-2023.

Ariño, A., Herrera, M., Juan, T., Estopañan, G., Carramiñana, J. J., and Rota, C. 2009. Influence of agricultural practices on the contamination of maize by fumonisin mycotoxins. J. Food Prot. 72:898-902.

Bihmidine, S., Hunter, C. T. III, Johns, C. E., Koch, K. E., and Braun, D. M. 2013. Regulation of assimilate import into sink organs: Update on molecular drivers of sink strength. Front Plant Sci. 4:177.

Blandino, M., and Reyneri, A. 2008. Effect of maize hybrid maturity and grain hardness on fumonisin and zearalenone contamination. Ital. J. Agron. 3:107-117.

Blandino, M., Reyneri, A., and Vanara, F. 2008. Influence of nitrogen fertilization on mycotoxin contamination of maize kernels. Crop Prot. 27:222-230.
Bolduan, C., Miedaner, T., Schipprack, W., Dhillon, B. S., and Melchinger, A. E. 2009. Genetic variation for resistance to ear rots and mycotoxins contamination in early European maize inbred lines. Crop Sci. 49: 2019-2028

Bommert, P., Nagasawa, N. S., and Jackson, D. 2013. Quantitative variation in maize kernel row number is controlled by the FASCIATED EAR2 locus. Nat. Genet. 45:334-337.

Boutigny, A.-L., Beukes, I., Small, I., Zühlke, S., Spiteller, M., and Van Rensburg, B. J. 2012. Quantitative detection of Fusarium pathogens and their mycotoxins in South African maize. Plant Pathol. 61:522-531.

Brown, P. J., Upadyayula, N., Mahone, G. S., Tian, F., Bradbury, P. J., and Myles, S. 2011. Distinct genetic architectures for male and female inflorescence traits of maize. PLoS Genet. 7:e1002383.

Busman, M., Butchko, R. A. E., and Proctor, R. H. 2012. LC-MS/MS method for the determination of the fungal pigment bikaverin in maize kernels as an indicator of ear rot. Food Addit. Contam. Part A. 29:1736-1742.

Clements, M. J., Kleinschmidt, C. E., Maragos, C. M., Pataky, J. K., and White, D. G. 2003. Evaluation of inoculation techniques for Fusarium ear rot and fumonisin contamination of corn. Plant Dis. 87:147-153.

Clements, M. J., Maragos, C. M., Pataky, J. K., and White, D. G. 2004. Sources of resistance to fumonisin accumulation in grain and Fusarium ear and kernel rot of corn. Phytopathology 94:251-260.

Costa, R. S., Môro, F. V., Môro, J. R., da Silva, H. P., and de Cássia Panizzi, R. 2003. Relação entre características morfológicas da cariopse e fusariose em milho [Relationship between caryopsis morphological characteristics and Fusarium ear rot in corn]. Pesqui. Agropecu. Bras. 38:27-33.

Desjardins, A. E., and Plattner, R. D. 1998. Distribution of fumonisins in maize ears infected with strains of Fusarium moniliforme that differ in fumonisin production. Plant Dis. 82:953-958.

Dombrink-Kurtzman, M. A., Dvorak, T. J., Barron, M. E., and Rooney, L. W. 2000. Effect of nixtamalization (alkaline cooking) on fumonisin-contaminated corn for production of masa and tortillas. J. Agric. Food Chem. 48:5781-5786.

Dowell, F. E., Pearson, T. C., Maghirang, E. B., Xie, F., and Wicklow, D. T. 2002. Reflectance and transmittance spectroscopy applied to detecting fumonisin in single corn kernels infected with Fusarium verticillioides. Cereal Chem. 79:222-226.

Duncan, K., and Howard, R. 2010. Biology of maize kernel infection by Fusarium verticillioides. Mol. Plant-Microbe Interact. 23:6-16.

Eller, M. S., Holland, J. B., and Payne, G. A. 2008. Breeding for improved resistance to fumonisin contamination in maize. Toxin Rev. 27:371-389.

Farrar, J. J., and Davis, R. M. 1991. Relationships among ear morphology, western flower thrips, and Fusarium ear rot of corn. Phytopathology 81: 661-666.

Fox, G., and Manley, M. 2009. Hardness methods for testing maize kernels. J. Agric. Food Chem. 57:5647-5657.

Horne, D. W., Eller, M. S., and Holland, J. B. 2016. Responses to recurrent index selection for reduced Fusarium ear rot and lodging and for increased yield in maize. Crop Sci. 56:85-94.

Humpf, H.-U., and Voss, K. A. 2004. Effects of thermal food processing on the chemical structure and toxicity of fumonisin mycotoxins. Mol. Nutr. Food Res. 48:255-269.

Jansen, C., Zhang, Y., Liu, H., Gonzalez-Portilla, P. J., Lauter, N., and Kumar, B. 2015. Genetic and agronomic assessment of cob traits in corn under low and normal nitrogen management conditions. Theor. Appl. Genet. 128: 1231-1242.

Kimanya, M. E., De Meulenaer, B., Roberfroid, D., and Lachat, C. 2010. Fumonisin exposure through maize in complementary foods is inversely associated with linear growth of infants in Tanzania. Mol. Nutr. Food Res. 54:1659-1667.

Lanubile, A., Maschietto, V., Borrelli, V. M., Stagnati, L., Logrieco, A. F., and Marocco, A. 2017. Molecular basis of resistance to Fusarium ear rot in maize. Front. Plant Sci. 8:1774.

Lazzaro, I., Busman, M., Battilani, P., and Butchko, R. A. E. 2012. FUM and $B I K$ gene expression contribute to describe fumonisin and bikaverin synthesis in Fusarium verticillioides. Int. J. Food Microbiol. 160:94-98.

Lorenz, A., Gustafson, T. J., Coors, J., and de Leon, N. 2010. Breeding maize for a bioeconomy: A literature survey examining harvest index and stover yield and their relationship to grain yield. Crop Sci. 50:1-12.

Lubin, J. H., Colt, J. S., Camann, D., Davis, S., Cerhan, J. R., and Severson, R. K. 2004. Epidemiologic evaluation of measurement data in the presence of detection limits. Environ. Health Perspect. 112:1691-1696.

Miller, J. D. 2001. Factors that affect the occurrence of fumonisin. Environ. Health Perspect. 109:321-324.

Mueller, D. S., Wise, K. A., Sisson, A. J., Allen, T. W., Bergstrom, G. C., Bosley, D. B. 2016. Corn yield loss estimates due to diseases in the United States and Ontario, Canada from 2012 to 2015. Plant Health Prog. 17: 211-222.

Munkvold, G. P. 2003. Cultural and genetic approaches to managing mycotoxins in maize. Annu. Rev. Phytopathol. 41:99-116. 
Munkvold, G. P., and Carlton, W. M. 1997. Influence of inoculation method on systemic Fusarium moniliforme infection of maize plants grown from infected seeds. Plant Dis. 81:211-216.

Munkvold, G. P., McGee, D. C., and Carlton, W. M. 1997. Importance of different pathways for maize kernel infection by Fusarium moniliforme. Phytopathology 87:209-217.

Mutiga, S. K., Were, V., Hoffmann, V., Harvey, J. W., Milgroom, M. G., and Nelson, R. J. 2014. Extent and drivers of mycotoxin contamination: Inferences from a survey of Kenyan maize mills. Phytopathology 104:1221-1231.

O'Brien, D., Yao, S., and Flores, A. 2010. Opening up a new world of maize. Agric. Res. 2010:4-6.

Parsons, M. W., and Munkvold, G. P. 2010. Associations of planting date, drought stress, and insects with Fusarium ear rot and fumonisin B1 contamination in California maize. Food Addit. Contam. 27:591-607.

Parsons, M. W., and Munkvold, G. P. 2012. Effects of planting date and environmental factors on Fusarium ear rot symptoms and fumonisin B1 accumulation in maize grown in six North American locations. Plant Pathol. 61:1130-1142.

Patience, J., and Ensley, S. 2010. Mycotoxin Contamination of Corn: What it is, what it does to pigs and what can be done about it. Online publication. Iowa State University Extension, Ames.

Pearson, T. C., Wicklow, D. T., and Brabec, D. L. 2009. Characteristics and sorting of white food corn contaminated with mycotoxins. Appl. Eng. Agric. 26:109-113.

Pearson, T. C., Wicklow, D. T., and Pasikatan, M. C. 2004. Reduction of aflatoxin and fumonisin contamination in yellow corn by high-speed dualwavelength sorting. Cereal Chem. 81:490-498.

Piepho, H.-P., and Moehring, J. 2007. Computing heritability and selection response from unbalanced plant breeding trials. Genetics 177:1881-1888.

Pollak, L. M. 2003. The history and success of the public-private project on germplasm enhancement of maize (GEM). Adv. Agron. 78:45-87.

Pollak, L. M., and Salhuana, W. 2001. The germplasm enhancement of maize (GEM) project: Private and public sector collaboration. Pages 319-329 in: Broadening the Genetic Base of Crop Production. H. D. Cooper, ed. CABI Publishing, Wallingford, Oxon, UK.

Presello, D. A., Botta, G., Iglesias, J., and Eyhérabide, G. H. 2008. Effect of disease severity on yield and grain fumonisin concentration of maize hybrids inoculated with Fusarium verticillioides. Crop Prot. 27:572-576.

Presello, D. A., Iglesias, J., Botta, G., and Eyhérabide, G. H. 2007. Severity of Fusarium ear rot and concentration of fumonisin in grain of Argentinian maize hybrids. Crop Prot. 26:852-855.

Rheeder, J. P., Marasas, W. F. O., and Vismer, H. F. 2002. Production of fumonisin analogs by Fusarium species. Appl. Environ. Microbiol. 68: 2101-2105.
Robertson, L. A., Kleinschmidt, C. E., White, D. G., Payne, G. A., Maragos, C. M., and Holland, J. B. 2006. Heritabilities and correlations of Fusarium ear rot resistance and fumonisin contamination resistance in two maize populations. Crop Sci. 46:353-361.

Robertson-Hoyt, L. A., Betrán, J., Payne, G. A., White, D. G., Isakeit, T., and Maragos, C. M. 2007. Relationships among resistances to Fusarium and Aspergillus ear rots and contamination by fumonisin and aflatoxin in maize. Phytopathology 97:311-317.

Robertson-Hoyt, L. A., Jines, M. P., Balint-Kurti, P. J., Kleinschmidt, C. E., White, D. G., and Payne, G. A. 2006. QTL mapping for Fusarium ear rot and fumonisin contamination resistance in two maize populations. Crop Sci. 46:1734-1743.

Rolletschek, H., Koch, K., Wobus, U., and Borisjuk, L. 2005. Positional cues for the starch/lipid balance in maize kernels and resource partitioning to the embryo. Plant J. 42:69-93.

Schjøth, J. E., Visconti, A., and Sundheim, L. 2009. Fumonisins in maize in relation to climate, planting time and hybrids in two agroecological zones in Zambia. Mycopathologia 167:209-219.

Shetty, P. H., and Bhat, R. V. 1999. A physical method for segregation of fumonisin-contaminated maize. Food Chem. 66:371-374.

Stasiewicz, M. J., Falade, T. D. O., Mutuma, M., Mutiga, S. K., Harvey, J. J. W., and Fox, G. 2017. Multi-spectral kernel sorting to reduce aflatoxins and fumonisins in Kenyan maize. Food Control 78:203-214.

USDA-FGIS. 2016. Grain Fungal Diseases and Mycotoxin Reference. Online publication. United States Department of Agriculture-Federal Grain Inspection Service, Washington, DC. www.gipsa.usda.gov/fgis/eBooks/ Mycobook/Fungal\%20Disease\%20and\%20Mycotoxin\%20Reference_2017. pdf

Venables, W., and Ripley, B. 2002. Modern Applied Statistics with S, 4th ed. Springer, New York.

Wild, C. P., and Gong, Y. Y. 2009. Mycotoxins and human disease: A largely ignored global health issue. Carcinogenesis 31:71-82.

Wild, C. P., Miller, J. D., and Groopman, J. D. 2015. Mycotoxin control in low- and middle-income countries. IARC Work. Group Rep. 9:1-53.

Zila, C. T., Ogut, F., Romay, M. C., Gardner, C. A., Buckler, E. S., and Holland, J. B. 2014. Genome-wide association study of Fusarium ear rot disease in the U.S.A. maize inbred line collection. BMC Plant Biol. 14:372.

Zila, C. T., Samayoa, L. F., Santiago, R., Butrón, A., and Holland, J. B. 2013. A genome-wide association study reveals genes associated with Fusarium ear rot resistance in a maize core diversity panel. G3 (Bethesda) 3: 2095-2104.

Zummo, N., and Scott, G. E. 1990. Cob and kernel infection by Aspergillus flavus and Fusarium moniliforme in inoculated, field-grown maize ears. Plant Dis. 74:627-631. 